Research Article

\title{
Optimal Design of the Six-Wheel Steering System with Multiple Steering Modes
}

\author{
Haixiang Bu $\mathbb{D},{ }^{1}$ Aijuan Li $\mathbb{D},{ }^{1,2}$ Xin Huang $\left(\mathbb{D},{ }^{3}\right.$ Wei Li $\left(\mathbb{D},{ }^{1}\right.$ and Jian Wang $\mathbb{C}^{1}$ \\ ${ }^{1}$ School of Automotive Engineering, Shan Dong Jiaotong University, Jinan 250357, China \\ ${ }^{2}$ Zhejiang Samsung Electromechanical Co. Ltd., Wenzhou 325000, China \\ ${ }^{3}$ School of Information Science and Electrical Engineering, Shan Dong Jiaotong University, Jinan 250357, China \\ Correspondence should be addressed to Aijuan Li; 65826990@qq.com and Xin Huang; huangxin@sdjtu.edu.cn
}

Received 20 May 2021; Accepted 26 November 2021; Published 27 December 2021

Academic Editor: Xianjian Jin

Copyright (C) 2021 Haixiang Bu et al. This is an open access article distributed under the Creative Commons Attribution License, which permits unrestricted use, distribution, and reproduction in any medium, provided the original work is properly cited.

Vehicles will face different working conditions during the use, and different working conditions have different requirements for vehicle functions, which results in many subdivided models. An off-road vehicle is a subdivision model produced to adapt to complex road conditions. In order to adapt to complex road conditions, vehicles should have a good passing ability, small size, and good flexibility. The six-wheeled vehicle has both good passing ability and small volume, which is the best choice for off-road vehicles. The design of the steering system becomes the key step to improve the flexibility of the six-wheeled vehicle. This paper mainly designs an independent steering system for a six-wheel electric vehicle with higher flexibility. The system is designed for six-wheel electric vehicles driven by six in-wheel motors. It mainly includes mechanical steering system and electronic control steering system. Both mechanical steering systems and electronic control steering system have multiple steering modes. Firstly, this paper introduces the various steering modes of the mechanical steering system and the electronic control steering system. Secondly, a CAD model is established by using the software Solidworks, and the system structure is introduced in detail combined with the CAD model. Finally, a kinematics model is established and calculated. The calculation results showed that the steering system can significantly improve the flexibility of the vehicle, so that the vehicle can complete the steering stably and quickly on complex road sections.

\section{Introduction}

With the rapid development of the automobile industry, automobile technology has made comprehensive progress. Among them, electric vehicles have developed rapidly with their excellent environmental performance, and the development of electric vehicles has alleviated the energy problem. In an electric vehicle, the steering system is one of the important subsystems, and the steering system has a great influence on the maneuverability and flexibility of the vehicle. Although the traditional steering system can achieve steering, in some cases, the vehicle cannot achieve steering easily. For example, the vehicle needs a small turning radius to pass through a narrow space or requires zero-radius steering to pass through a curve with a large turn angle. With the rapid development of the automobile industry, automobile practitioners have proposed several new types of steering systems, which improve the flexibility of vehicles. Relevant documents introduced a new type of electric vehicle steering system, including four-wheel steering system [1-4], in-wheel motor drive system [5], control of steering system [6-10], kinematics model, and calculation of four-wheel steering [11-13]. The above system is applied to the four-wheel steering system to improve the handling and flexibility of the four-wheel steering vehicle. The four-wheel steering system includes a four-wheel independent steering system and a four-wheel linkage steering system. The four-wheel independent steering system generally uses the steering motor to drive the wheels to steer independently, so that the steering angle of each wheel can be controlled separately, which improves the flexibility of the vehicle, but the stability is relatively poor. The four-wheel linkage steering system is 
generally mechanical linkage steering. In this way, the fourwheel linkage steering has better stability but less steering modes that can be achieved, and the vehicle's flexibility is relatively poor. Therefore, a steering system that combines mechanical steering and electronic control steering is needed to achieve the combination of stability and flexibility.

However, four-wheeled vehicles have a poor passing ability when passing through potholes. In order to improve the passing ability of the vehicle on the complex roads, it is necessary to increase the number of axles. And the length of the four-axle and the eight-wheeled vehicle is too large, which is not conducive to passing through narrow sections during off-road conditions. The three-axle six-wheel vehicle not only has a strong passing ability when passing potholes but also can ensure that its vehicle length is short enough to pass through narrow sections. It is the best choice of axle number for off-road vehicles. Based on the above reasons, this paper proposes a new type of steering system. This steering system is a steering system designed for a three-axle six-wheel in-wheel motor-driven vehicle, which realizes the combination of mechanical steering and electronic control steering. The vehicles driven by in-wheel motors can achieve greater steering angles, and the vehicles can achieve more steering modes. Due to the adoption of a new steering structure, this system has the following advantages:

(1) It can realize multiple steering modes such as the front two-wheel steering mode, the six-wheel inphase steering mode, the six-wheel reverse-phase steering mode, lateral parking mode, and the zeroradius steering mode. The multiple steering modes make the steering system more flexible.

(2) The electronic control six-wheel steering system has high flexibility, and the mechanical six-wheel steering system has high stability. This steering system realizes the combination of the electronically controlled six-wheel steering system and the mechanical six-wheel steering system so that the system has both the advantages of the electronic control sixwheel steering system and the advantages of the mechanical six-wheel steering system, which makes the steering system have high flexibility and high stability.

(3) The mechanical steering system and the electronic control steering system can work independently. Without the electronic control steering system, the mechanical steering system can realize the three steering modes of the front two-wheel steering, sixwheel in-phase steering, and six-wheel reverse-phase steering. The mechanical steering system can ensure the stable operation of the steering system. The electronic control steering system can also work independently without the use of a mechanical steering system. The electronic control steering system can realize five steering modes to improve the flexibility of the vehicle.
(4) The electronic control steering system can execute the steering command issued by the automatic driving module to realize the steering of automatic driving. Thanks to the multiple steering modes of the system, the driver or the automatic driving module can select the steering mode to control the vehicle, so that the vehicle can adapt to different road conditions with a more appropriate steering mode, and improve the flexibility and passing ability of the vehicle.

The first section of this paper introduces the steering modes of the system $[14,15]$ and introduces the application scenarios of each steering mode. In the second section, the six-wheel steering system structure is described, and its main components are introduced briefly. The third section combines the CAD model to introduce the working principle of the steering system components in detail and explains the switching principle of the mechanical steering mode of the system. The fourth section mainly introduces the electronic control steering system including system hardware and control methods. The fifth section calculates the steering system, which verifies the performance of the system and provides the necessary data for the electronic control system.

\section{Steering Mode and Its Application}

This system is a steering system of a six-wheel electric vehicle driven by six in-wheel motors independently. Due to the use of the in-wheel motor drive, the vehicle can achieve largeangle steering easily, which can also achieve more steering modes. Moreover, this steering system adopts a combination of mechanical steering and electronic control steering. Both the mechanical steering system and the electronic control steering system have multiple steering modes.

Figure 1 is a schematic diagram of the steering mode, where Figure 1(a) indicates the vehicle is not steering, Figure 1(b) indicates the front two-wheel steering mode, Figure 1(c) indicates the six-wheel reverse-phase steering mode, Figure 1(d) indicates the six-wheel in-phase steering mode, Figure 1(e) indicates the zero-radius steering mode, and Figure 1(f) indicates the lateral parking mode. Figure 2 is a schematic diagram of the application scenario of the steering mode, where Figure 2(a) represents the application of the six-wheel reverse-phase steering mode, Figure 2(b) represents the application of the six-wheel in-phase steering mode, Figure 2(c) represents the application of the zero-radius steering mode, and Figure 2(d) represents the application of the lateral parking mode, because the application of the front two-wheel steering mode is relatively simple, so it is not marked in Figure 2. The steering mode is introduced in combination with Figure 1, and the application of steering mode is introduced in combination with Figure 2. 


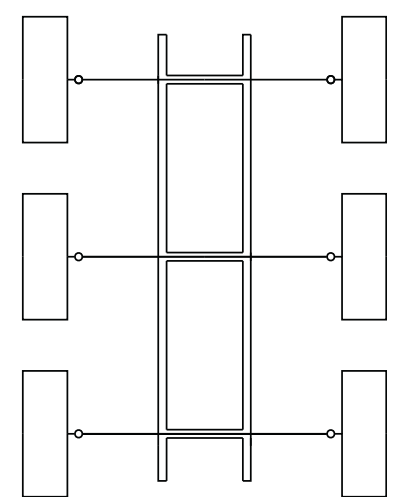

(a)

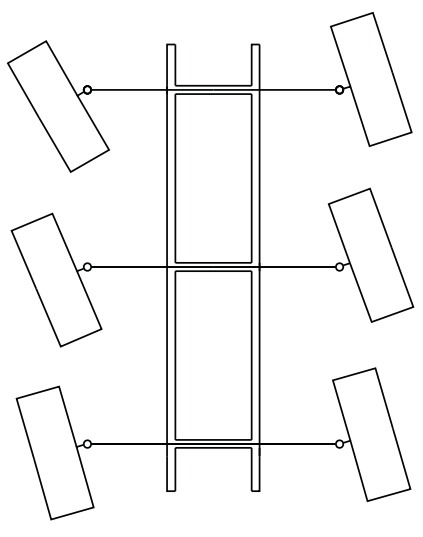

(d)

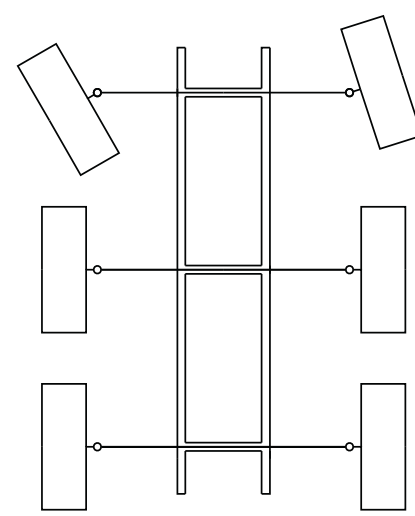

(b)

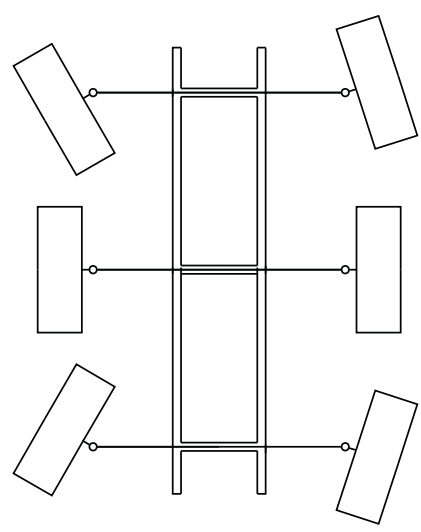

(c)

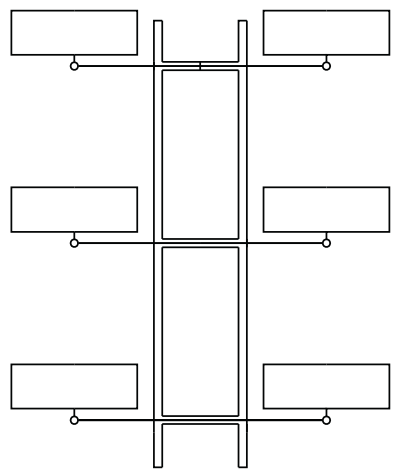

(e)

(f)

Figure 1: Schematic diagram of steering mode.

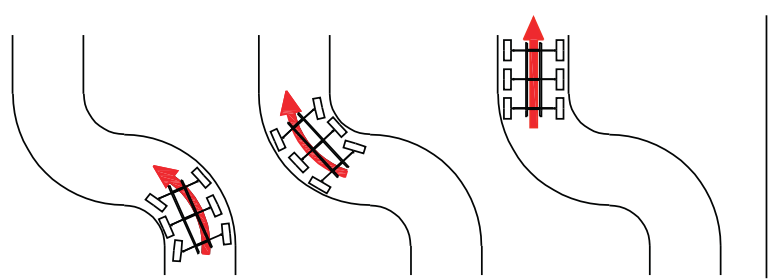

(a)
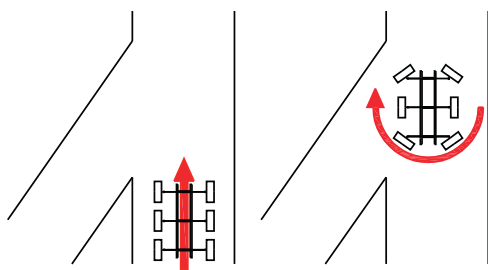

(c)
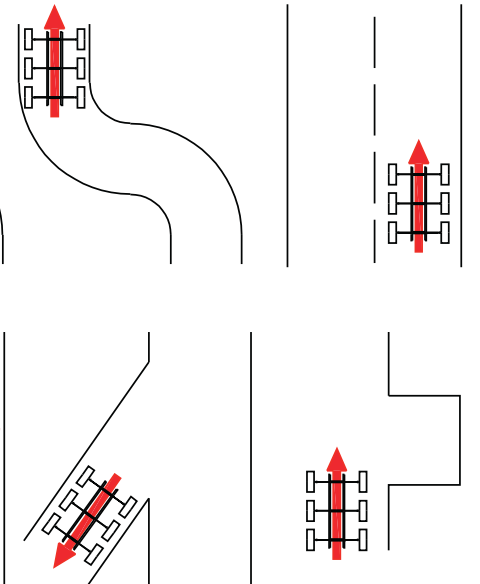
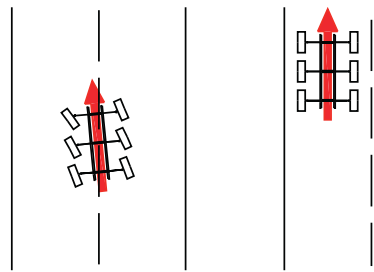

(b)
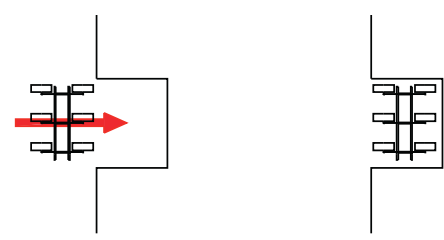

(d)

FIgURE 2: Schematic diagram of the application scenario of the steering mode.

2.1. Front Two-Wheel Steering. When the steering system is in the mechanical front two-wheel steering mode, the mechanical steering mechanism of the rear four wheels is locked. Only the front-wheel steering mechanism is linked with the steering wheel, and the steering operation is relatively light at this time, which can reduce the labor intensity of the driver. When the front two wheels are steered electronically, only the steering motors of the front two wheels need to be controlled independently, and the control strategy is relatively simple. The vehicle can adopt front twowheel steering mode when driving at medium or low speeds on a generally straight road. 
2.2. Six-Wheel Reverse-Phase Steering. When the steering system is in the mechanical six-wheel reverse-phase steering mode, the steering wheel is linked with the steering mechanism of the first and third axle. At this time, the mechanical steering mechanism of the intermediate axle is locked, and the steering gears of the first and third axles are linked reversely. When in the electric control six-wheel reverse-phase steering mode, the four steering motors of the first axle and the third axle drive the four wheels to steer independently. The six-wheel reverse-phase steering mode has a smaller steering radius and better flexibility. As shown in Figure 2(a), the six-wheel reverse-phase steering is suitable for working conditions when steering in a narrow space such as an S-shaped curve. Even in a narrower and more tortuous S-shaped curve, the vehicle can pass quickly.

2.3. Six-Wheel In-Phase Steering. When the steering system is in the six-wheel in-phase steering mode, all six wheels steer in the same direction, which can ensure that the tires receive less lateral force when the vehicle is steering, and increase the steering stability of the vehicle. When the steering system is in the mechanical six-wheel in-phase steering mode, the steering wheel and the three axles are linked. When the steering system is in the electronic control six-wheel in-phase steering mode, the steering controller controls the six steering motors independently, and the motors drive the six wheels to steer independently. This mode is suitable for high-speed vehicle operating conditions. As shown in Figure 2(b), when the vehicle changes lanes at high speed, the six wheels steer at the same time, which makes the vehicle more stable and has better maneuverability.

2.4. Zero-Radius Steering. The mechanical steering system of this steering system cannot realize the function of zeroradius steering, and the zero-radius steering mode can only be realized by an electronic control steering system. In the zero-radius steering mode, the steering center of the vehicle is located at the center of the second axle, and the wheels of the second axle do not steer when the vehicle is steering. The steering controller controls the four steering motors of the first and third axles to drive the wheels to steer independently. The zero-radius steering mode is mainly used to realize a zero-radius U-turn of the vehicle and is suitable for steering the vehicle in a narrow space. As shown in Figure 2(c), when a vehicle passes a Y-shaped intersection in a narrow space, it can turn directly to the target direction by zero-radius steering mode, so that the vehicle can pass the intersection quickly.

2.5. Lateral Parking. The mechanical steering system of this steering system cannot realize the function of lateral parking, and the lateral parking mode can only be realized by the electronic control steering system. When moving laterally, the steering controller controls the six motors to drive the six wheels to rotate $90^{\circ}$ independently. At this time, the wheels are parallel to the $Y$ direction of the vehicle coordinate system, and the vehicle can move laterally easily. The main design purpose of lateral parking is to facilitate parking on the side of the city. As shown in Figure 2(d), the lateral parking space is only slightly larger than the vehicle. If it is a car equipped with a traditional steering mechanism, it cannot park in the parking space. However, the steering mode of lateral movement allows the vehicle to move laterally into the parking space directly, making the parking of the vehicle more convenient.

\section{System Structure}

The advantage of traditional mechanical steering technology is to maintain kinematic constraints through the mechanical connecting rods of the front wheels. The traditional vehicle steering system has good stability, which can ensure the safety of vehicle driving. However, in order to achieve zeroradius steering and lateral parking, each wheel needs to steer independently, and each wheel is equipped with an independent steering motor, which can realize the independent steering of the wheel. The independent steering system of the wheels has better functionality, but its control is more difficult. Although each wheel can achieve the kinematic constraint in a steady state independently, it can not guarantee the kinematic constraint in a transient state. Based on the problems of the traditional vehicle steering system and independent steering system, a new steering system is proposed in this paper. The system is composed of a mechanical steering system and electric control steering system. The system combines the advantages of a mechanical steering system and electric control steering system and has good stability and function. Both the mechanical steering system and electric steering system have many steering modes, which can choose more appropriate steering mode in different scenarios.

The mechanical steering system of this system is pure mechanical steering in essence. The mechanical steering system mainly includes the steering wheel, the transmission device of the steering wheel and the steering mechanism, the steering mechanism on the three axles, and the longitudinal linkage rack. The driver controls the steering system through the steering wheel, and the transmission device of the steering wheel and the steering mechanism can transmit the steering wheel angle to the steering mechanism of the first axle. The steering mechanism of the first axles is linked with the steering mechanism of the other two axles through the longitudinal transmission rack to realize the three-axle linkage steering. The longitudinal movement of the longitudinal transmission rack can realize the linkage of three steering mechanisms, and the lateral movement of the longitudinal transmission rack can switch the steering mode.

The electronic control steering system and the mechanical steering system can work independently, but they are not two completely independent systems in the design scheme, and some parts are shared. The electronic control steering system also drives the wheels to steer through the tie rod, and the steering motor is installed on the sliding base. When the steering motor drives the tie rod to achieve steering, the sliding base is fixed, and each sliding base is 
equipped with two steering motors, and the steering motor drives the corresponding wheels to steer independently. And when the motor drives the tie rod to move, the screw transmission mechanism is adopted, which makes it produce a self-locking effect. In this way, the steering motor can drive the tie rod to move, but the tie rod cannot drive the steering motor to move, which improves the accuracy of motor control.

\section{CAD Modeling}

The structure of the steering system is relatively complicated. In order to analyze the steering system comprehensively, this paper uses the software Solidworks to establish a three-dimensional CAD model. Figure 3 is the overall model of the steering system. From the figure, the overall structure of the steering system can be seen. The following is a detailed introduction of this structure combined with a three-dimensional CAD model.

4.1. Steering Mechanism. The steering system has three steering mechanisms; each axle has a steering mechanism. The structures of the steering mechanism on the three axles are roughly the same. However, in order to realize different functions of the steering mechanism on each axle, the parts on the steering mechanism are different to a certain extent. The sliding sleeve of the steering mechanism of the first axle is connected with the transmission shaft, the steering mechanism of the second axle needs to have a locking device, and the steering mechanism of the third axle needs to have a gear locking device and a reverse steering function. The following takes the steering mechanism of the second axles as an example to introduce. As shown in Figures 4 and 5, it includes a sliding base, a fixed base, a sliding sleeve, a gear set, and a steering rack.

The composition of the sliding base is shown in Figures 6 and 7. Two steering motors are fixed on the sliding base, and the steering motor drives the driving gear and the driven gear to rotate. There is a threaded hole in the center of the driven gear, the $\mathrm{D}$-type screw is matched with the threaded hole of the driven gear. The axial displacement of the driven gear is fixed and can only be rotated. Then, the D-type screw and the driven gear form a spiral transmission mechanism. In this way, the rotation of the motor can be converted into the lateral movement of the D-type screw to drive the wheels to steer, and the screw transmission mechanism can be selflocking, so the D-type screw cannot drive the motor in reverse, which reduces the difficulty of controlling the motor, and also improves control precision. In the electronic control steering mode, the sliding base is in the initial position and locked. At this time, the sliding base cannot slide. The two steering motors drive the D-type screw to move independently, thereby driving the tie rod to achieve steering. In mechanical steering mode, since the screw mechanism can be self-locked, the D-type screw will not move relative to the sliding base when the steering motor is not working, so the D-type screw and the sliding base are relatively fixed, and the movement of the sliding base can directly drive the tie rod to realize steering.
4.2. Linkage Mode of Steering Mechanism. This system can realize six-wheel steering, and six-wheel steering needs to realize the linkage of the steering mechanism on the three axles. The steering mechanism linkage diagram is shown in Figure 8. The longitudinal linkage rack realizes the linkage of the steering mechanism on the three axles. The longitudinal linkage rack has longitudinal and lateral movement relative to the fixed base. The longitudinal movement of the longitudinal linkage rack will drive the steering mechanism to linked steering, and the lateral movement can switch the steering mode. The installation of the longitudinal linkage rack and the sliding sleeve is shown in Figure 9. This paper uses the sliding sleeve to connect the longitudinal linkage rack and the fixed base. The sliding sleeve can slide left and right within a certain range on the fixed base, and the longitudinal linkage rack can move longitudinally relative to the sliding sleeve. There is a linkage gear set on the fixed base, and the linkage gear set has a driving gear, which drives the steering rack to move when the driving gear rotates. The steering rack drives the sliding base to move left and right, which drives the wheels to steer. The longitudinal linkage rack meshes with the driving gear on the three steering gears to realize the linkage of the steering gear, and the meshing situation varies according to the steering mode.

4.3. Switching the Steering Mode. This system has two steering modes: the mechanical steering mode and the electronic control steering mode. The conversion of electronic control steering mode can be realized in the control strategy. The switch of mechanical steering mode will be introduced first. For the stability of mechanical steering mode switching, there is an initial state of the mechanical steering system. The initial state of mechanical steering is that all wheels are not steering. The steering system needs to be in the initial state when switching the steering mode.

The meshing conditions of the longitudinal linkage rack and the driving gears on the three steering mechanisms are different under different steering modes. The longitudinal linkage rack changes the meshing with the driving gear through lateral movement and realizes the conversion of the steering mode. The switching of the steering mode is shown in Figure 10. Figure 10(a) is the front two-wheel steering mode. In this mode, only the steering gear of the first axle meshes with the longitudinal linkage rack. Figure 10(b) is a six-wheel reverse-phase steering mode. In this mode, the steering gear of the first axle and the reverse-phase steering gear of the third axle mesh with the longitudinal linkage rack. Figure 10(c) is the six-wheel in-phase steering mode. In this mode, the steering gear of the first axle, the steering gear of the second axle, and the in-phase steering gear of the third axle mesh with the longitudinal linkage rack. When the gear and the rack are disengaged, there is a corresponding mechanism to lock it, so that the next time it is engaged, it can be engaged in the correct position. The mode switching joystick drives the sliding base to move by pulling the wire, thereby driving the longitudinal linkage rack to move horizontally. The parts in Figure 10 are only schematic diagrams and do not reflect the size of the parts. 


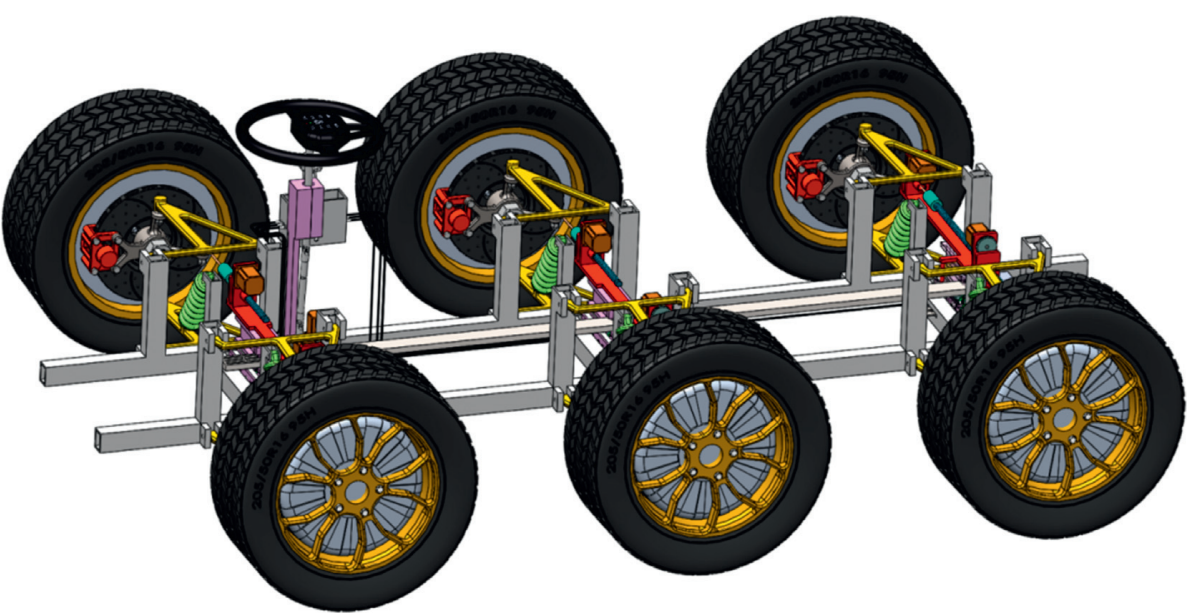

Figure 3: General diagram of the steering system.

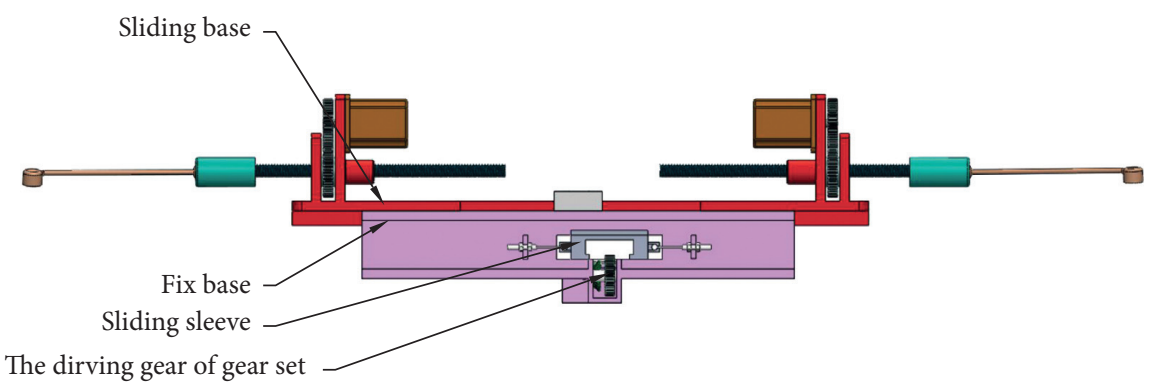

FIgURE 4: First view of the steering gear.

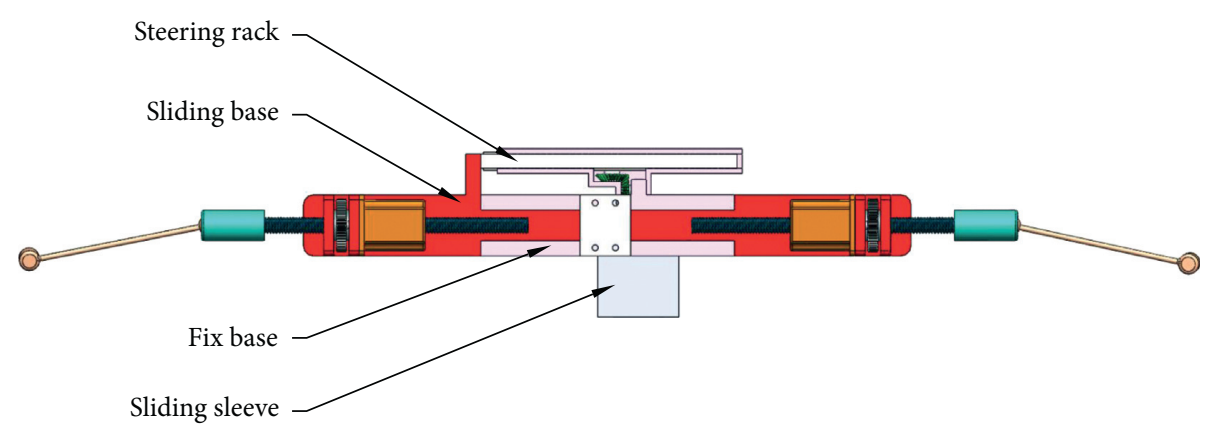

Figure 5: Second view of the steering gear.

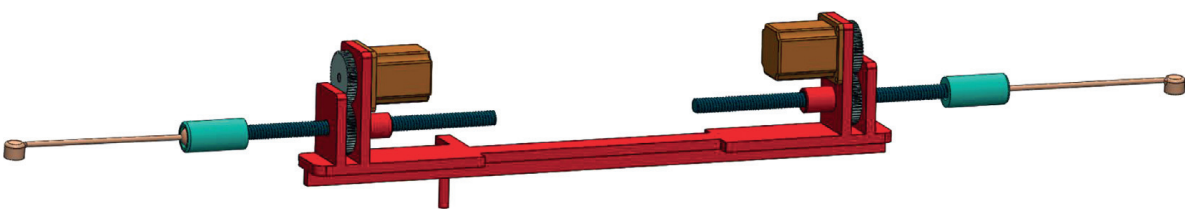

Figure 6: General composition of the sliding base.

4.4. Transmission of Steering Wheel and Longitudinal Linkage Rack. The steering wheel drives the longitudinal movement of the longitudinal linkage rack to drive the corresponding sliding base to move to achieve steering. The schematic diagram of the steering wheel and longitudinal linkage rack transmission is shown in Figure 11. The steering wheel drives the steering column, the universal joint, the transmission shaft, the gear set on the sliding sleeve, and finally the pinion of the gear set to rotate. The pinion meshes in the longitudinal groove of the longitudinal linkage rack. The gear set on the sliding sleeve is fixed on the sliding sleeve of the first axle, and the sliding sleeve can only slide laterally, so when the pinion 


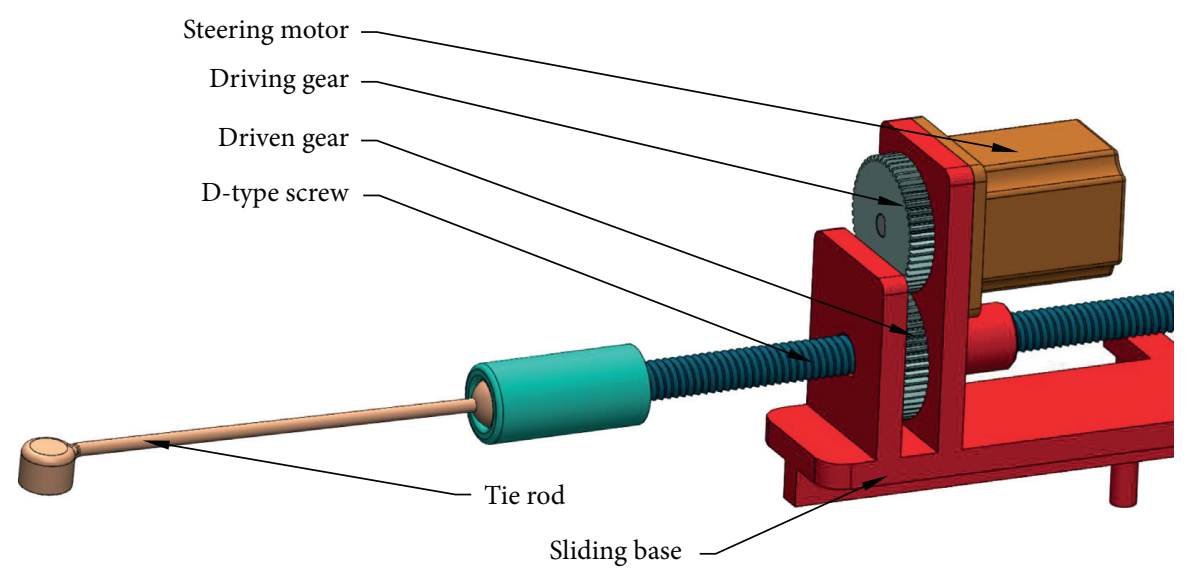

FIGURE 7: Enlarged view of sliding base.

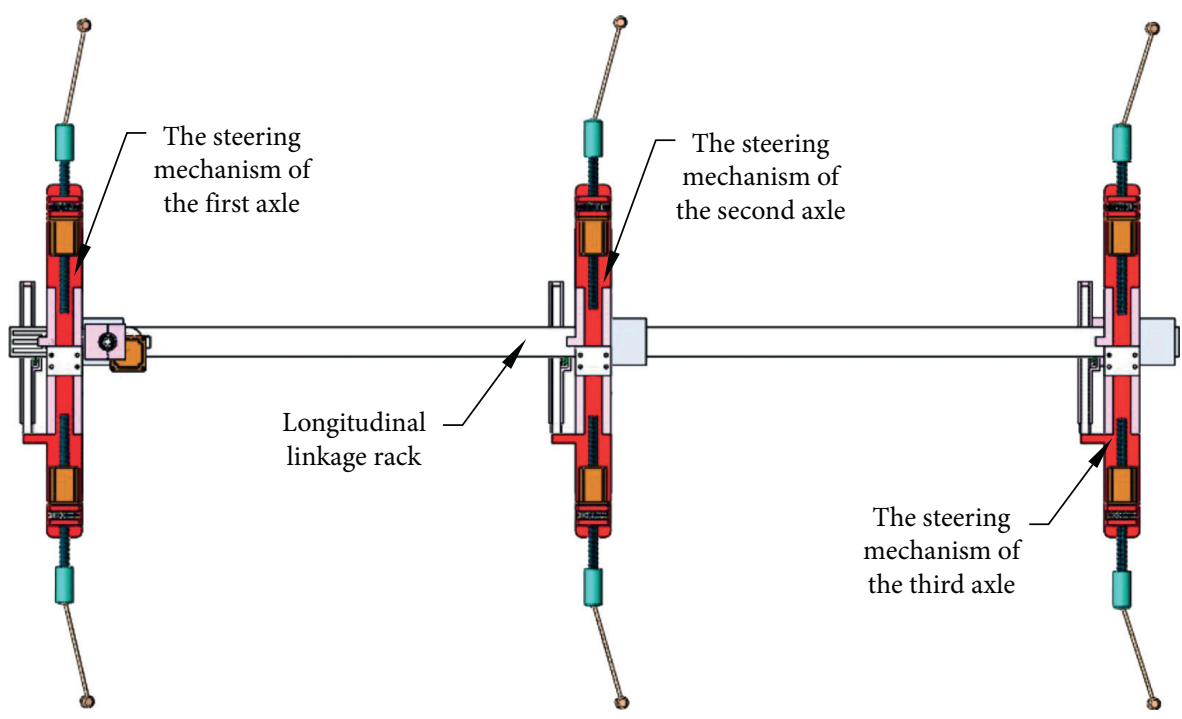

FIGURE 8: Steering mechanism linkage diagram.

rotates, it drives the longitudinal linkage rack to move longitudinally and will not cause lateral movement of the sliding sleeve. The power-assisted motor in Figure 11 plays the role of assisting the driver when steering and restoring the initial state of mechanical steering when the electronic control mode is activated.

\section{Control of the Electronic Steering System}

5.1. Operation of Electronic Steering. The operation of the electronic control steering system can be controlled directly by the driver, and it can also be controlled by the automatic driving module. The automatic driving module does not need to operate the panel, and the driver needs to have an operation panel when operating, and the operation panel of the steering system is integrated into the steering wheel. The steering wheel control panel is shown in Figure 12.

The power button is used to activate and deactivate the electronic control steering system. In Figure 12, the five buttons 2FWS, 6WRS, 6WIS, LP, and ZRS are the selection buttons for the five steering modes of the front two-wheel steering mode, six-wheel reverse-phase steering mode, sixwheel in-phase steering mode, lateral shift parking mode, and zero-radius steering mode. The two buttons of the left and right arrows are used to control the steering direction, and the reset button is used to reset the electronic control steering system.

The driver presses the power button for 3 seconds to enter the electronic control mode. After the green light flashes, press the corresponding steering mode button to select the steering mode. After the green light is always on, it enters the corresponding steering mode. At this time, pressing the two buttons of the left and right arrows will turn the steering system in the corresponding direction and release the button to stop the steering system.

5.2. Realization of Control. In order to realize the functions of this system, the hardware of the electrical system needs to cooperate to realize cooperative work. Figure 13 is the hardware diagram of this system. The system hardware needs a corresponding control method to realize the control 


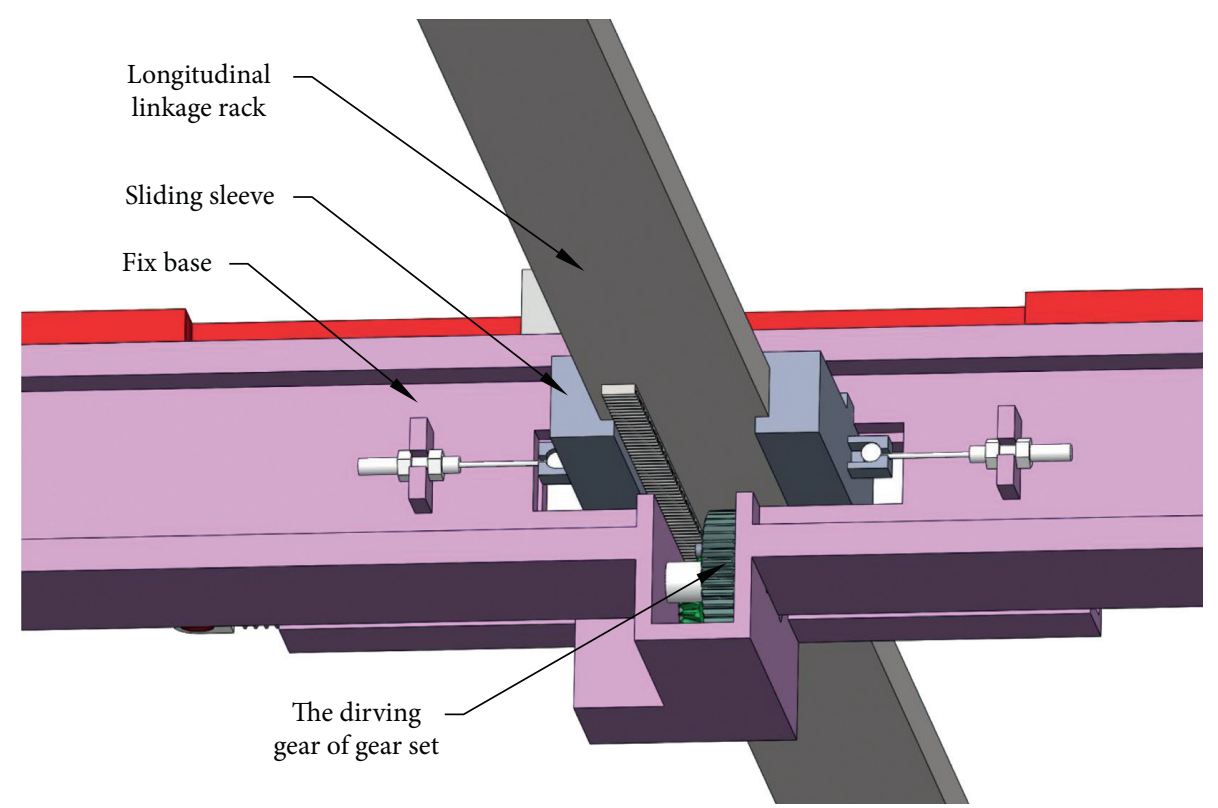

FIGURE 9: Installation diagram of the sliding sleeve of longitudinal linkage rack.

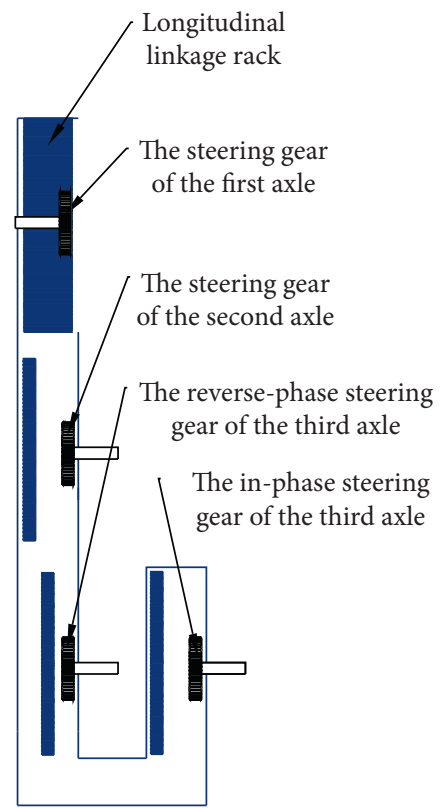

(a)

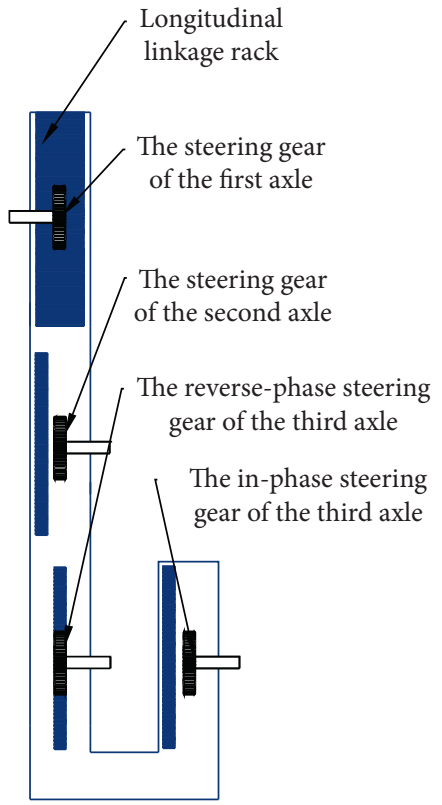

(b)

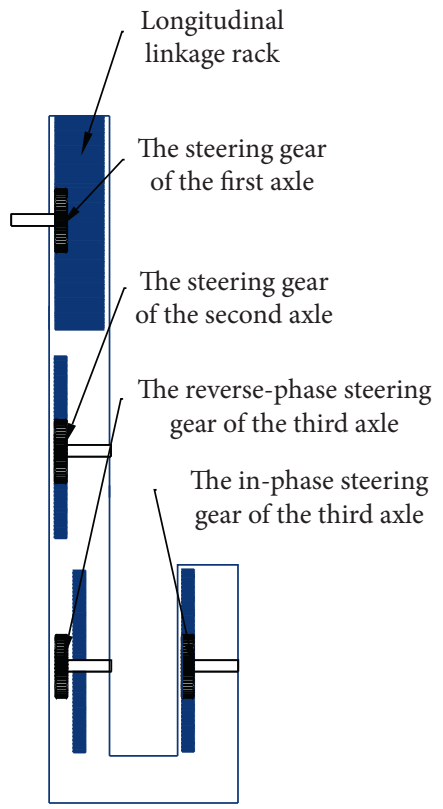

(c)

FIgURE 10: Schematic diagram of steering mode.

of the system. The control method of this system will be introduced combined with the system hardware.

In the default state, the electronic control steering system is not started. When entering the electronic control steering mode, the electronic control steering system should be started first. Firstly, when starting the electronic control steering system, the steering controller will receive the signal of starting the electronic control steering system from the control button or the automatic driving module. After receiving this signal, the wheel angle sensor will detect whether the wheel is in the initial steering state. If it is in the initial steering state, the mechanical steering locking motor will lock the mechanical steering system. If it is not in the initial steering state, the red light on the control panel will flash, and then the power-assisted motor will restore the steering system to the initial steering state. The red light no longer flashes after returning to the initial state. At this time, the locking motor of the mechanical steering will lock the mechanical steering system, and the green light will flash after the mechanical steering system is locked. Then, the corresponding steering mode is selected according to the steering mode signal input by the automatic driving module 


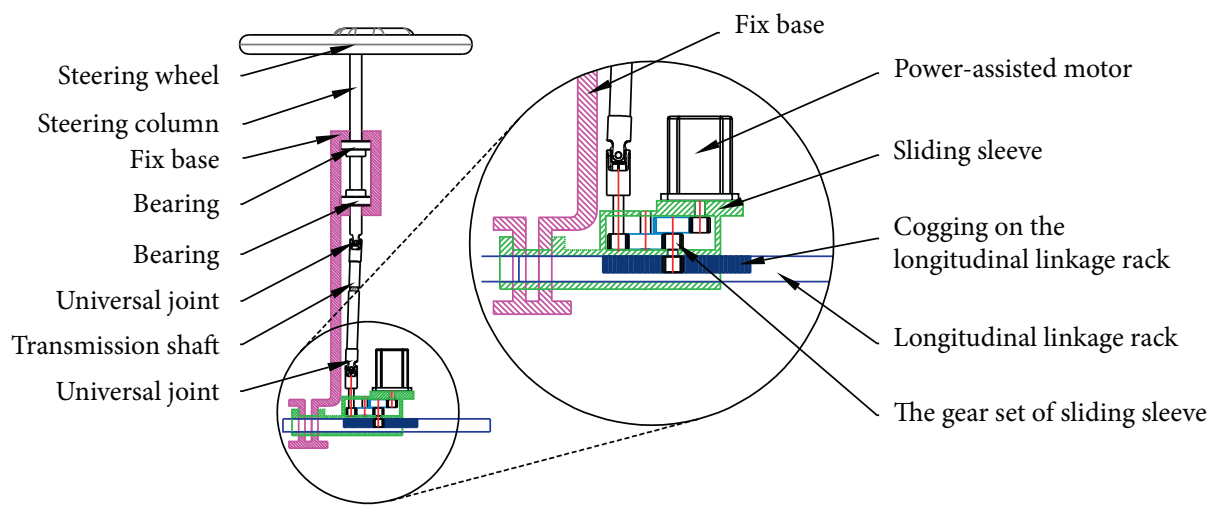

FIGURE 11: Schematic diagram of the steering wheel and longitudinal linkage rack transmission.

or the driver. After confirming the steering mode, the green light is always on, and the steering system enters the electronic control steering state.

The control input of the system has two input modes: the input of the automatic driving module and the input of the driver. When the automatic driving module inputs the control signal, the automatic driving controller analyzes the data collected by the automatic driving sensor and then calculates the steering angle of the front inner wheel and then inputs the steering angle signal of the front inner wheel to the steering controller. When the driver inputs the control signal, the driver inputs the front inner wheel angle signal to the steering controller by pressing the button. After the steering controller receives the input signal of the front inner wheel angle, it compares the steering angles of the remaining five wheels in the database according to the steering mode and then controls the steering motor separately according to the steering angle of each wheel. After the steering motor generates the steering angle, the wheel angle sensor detects the wheel angle, corrects the error of the angle in time, and realizes the closed-loop control of the steering angle. The steering controller individually controls the steering angle of each steering motor to realize the independent steer of the wheels by a certain angle. In order to satisfy Ackerman's theorem $[16,17]$ under different steering modes, the steering angle of each wheel is different. In the steering kinematics model, the relationship between the wheel steering angles in various modes is calculated.

\section{Modeling and Analysis}

When a vehicle is steering, it will be affected by the tire slip angle, and the tire slip angle affects the vehicle's steering center. Since there are many factors that affect the tire slip angle, and it is difficult to accurately determine, the following is to analyze the steering problem of the vehicle under the influence of ignoring the slip angle. Since the vehicle has different steering modes, this section will analyze the different steering modes and compare the steering states under different steering modes. steering system has five steering modes, including the front two-wheel steering mode, six-wheel reverse-phase steering mode, six-wheel in-phase steering mode, and zero-radius steering mode, which are all steering around a steering center. This paper mainly analyzes the kinematics of these four steering modes. In the lateral parking mode, the vehicle moves horizontally, and the kinematics model is relatively simple, so the kinematics analysis is not needed.

(1) Front two-wheel steering mode: for the general steering system, the axes of all wheels should intersect at the instantaneous steering center. The system has three axles, and the wheels of the rear two axles do not steer in this mode. In this mode, not all the axes of the wheels intersect at the instantaneous steering center, and the instantaneous steering center is located in the middle of the rear two axles.

As shown in Figure 14, the inner wheel steering angle of the first axle is $\alpha_{1}$, the outer wheel steering angle of the first axle is $\beta_{1}$, the wheelbase $L_{1}=1350 \mathrm{~mm}$, and the kingpin distance $K=1225 \mathrm{~mm}$. Usually, the maximum steering angle of the wheel is $35^{\circ}$; although the system can achieve a large steering angle, it cannot make the steering angle too large in this mode. The maximum wheel's steering angle of the system is controlled at $35^{\circ}$, and the minimum steering radius is calculated.

When the minimum turning radius is calculated, the outer wheel steering angle is required. And when the vehicle is steering, the inner wheel steering angle is greater than the outer wheel steering angle. Therefore, the steering angle of the inner wheel is limited by a maximum of $35^{\circ}$. In order to obtain the degree of outer wheel steering angle when the inner wheel steering angle is $35^{\circ}$, the relationship between the steering angle of the inner wheel and the steering angle of the outer wheel is required. The relationship between the inner wheel steering angle $\alpha$ and the outer wheel steering angle $\beta$ is as follows:

$$
\cot \beta-\cot \alpha=\frac{K}{L}
$$

According to formula (1), the calculation formula of $\beta$ is obtained: 


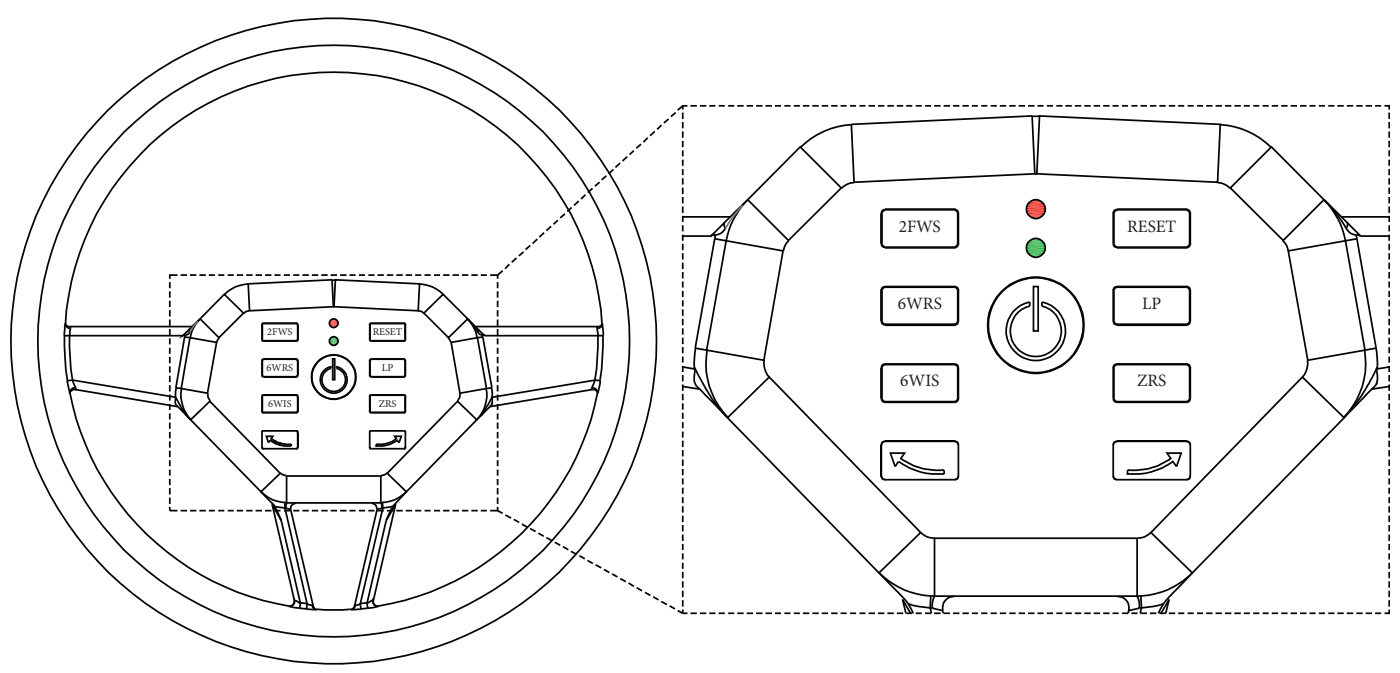

FIGURE 12: Steering wheel control panel.

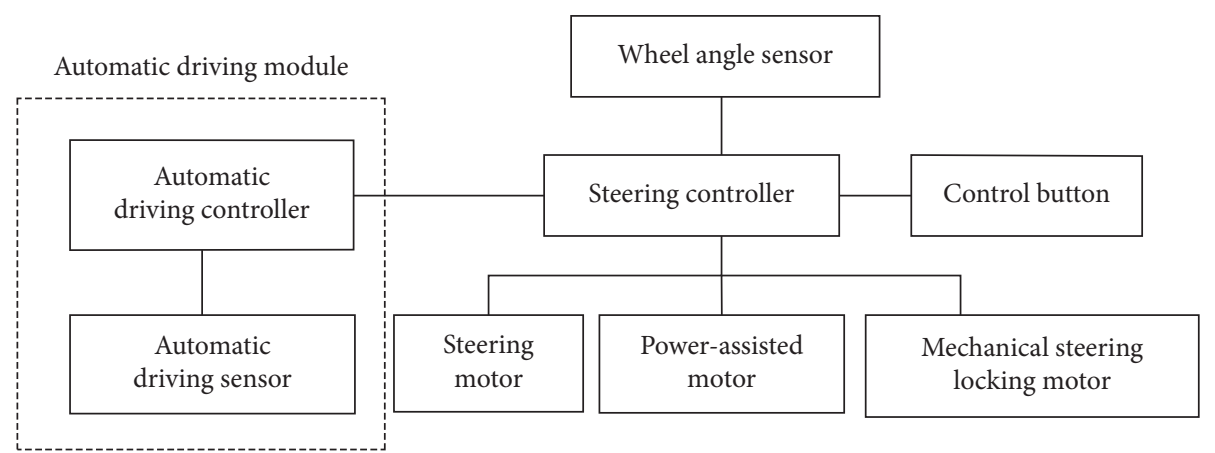

FIgURE 13: Hardware diagram of the electronic control steering system.

$$
\beta=\arctan \left(\frac{1}{(K / L)+(1 / \tan \alpha)}\right) .
$$

And, the steering radius of the vehicle can be calculated by

$$
r=\frac{L}{\sin \beta}+r_{S} .
$$

When formula (2) is brought into formula (3), we can get the relationship between $r$ and $L, K, \alpha$, and $r_{S}$ which is shown in

$$
r=\frac{L}{\sin (\arctan (1 /((K / L)+(1 / \tan \alpha))))}+r_{S} .
$$

From formula (1) to formula (4), $r$ is the steering radius, $\beta$ is the outer wheel steering angle, $\alpha$ is the inner wheel steering angle, $K$ is the kingpin distance and $K=1225 \mathrm{~mm}, r_{S}$ is the kingpin offset distance, and $r_{S}=165 \mathrm{~mm}$. For general vehicles, $L$ is the wheelbase, but for multiaxle vehicles, $L$ is the distance from the steering axle to the horizontal straight line where the steering center is located. So, in this system, $L$ is different in different steering modes.
In the front two-wheel steering mode, the relationship between the inner wheel steering angle $\alpha_{1}$ and outer wheel steering angle $\beta_{1}$ of the first axle can be calculated according to formula (2), and the result is shown in Figure 15. According to formula (4), the turning radius can be calculated. And in the front two-wheel steering mode, the distance from the steering axle to the horizontal straight line where the steering center is located is $L_{1}$, so in this mode, $L=L_{1}=1225 \mathrm{~mm}$. When the inner wheel steering angle is the maximum, the steering radius is the minimum, and the maximum inner wheel steering angle is $35^{\circ}$. Putting $L=1350 \mathrm{~mm}, \quad \alpha=35^{\circ}$, $K=1225 \mathrm{~mm}$, and $r_{S}=165 \mathrm{~mm}$ into formula (4), we can calculate the minimum turning radius, and the minimum steering radius in front two-wheel steering mode is about $3595 \mathrm{~mm}$ calculated by formula (4).

(2) Six-wheel reverse-phase steering mode: in this mode, the steering center is on the extension line of the second axle, the wheels of the second axle do not steer in this mode, and the wheels of the first and third axles steer.

As shown in Figure 16, the inner wheel steering angle of the first axle is $\alpha_{2}$, the outer wheel steering angle of 


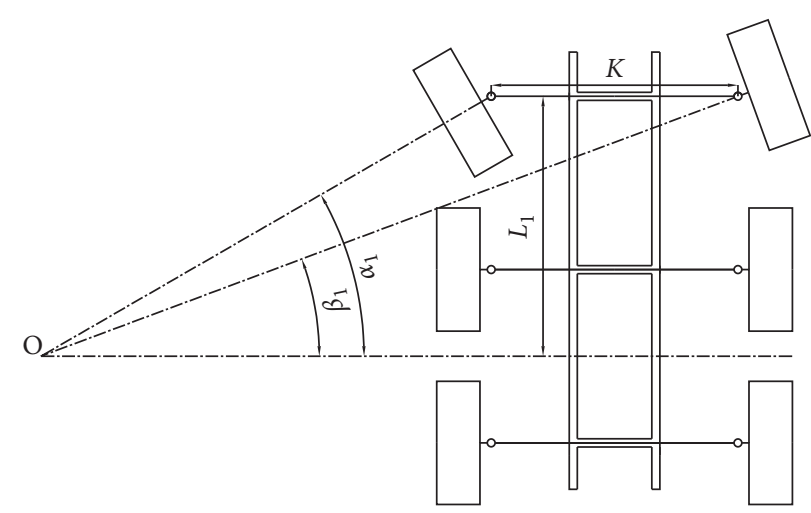

FIgURE 14: Schematic diagram of the steering angle of each wheel in the front two-wheel steering mode.

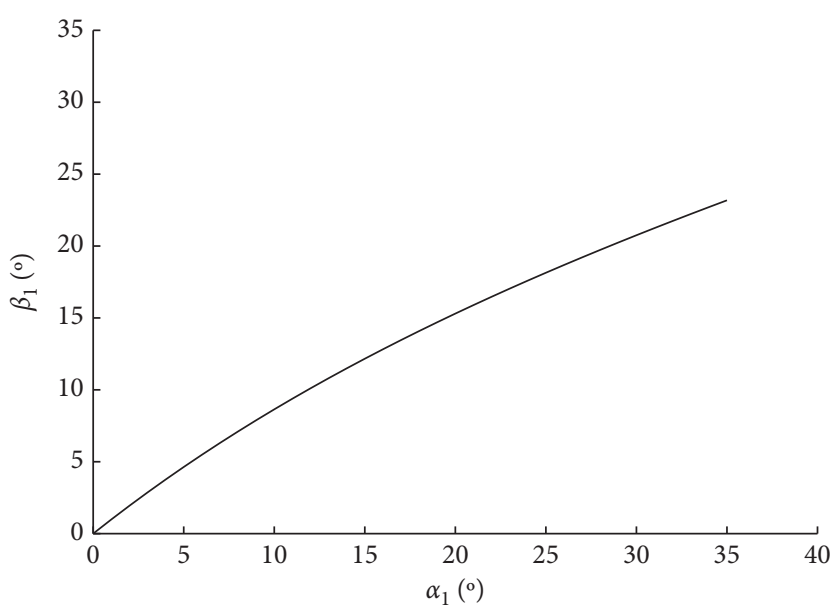

FIGURE 15: The relationship diagram of the first axle inner wheel angle for the outer wheel angle.

the first axle is $\beta_{2}$, the inner wheel steering angle of the third axle is $\alpha_{3}$, the outer wheel steering angle of the third axle is $\beta_{3}$, the wheelbase between the first axle and the second axle is $L_{2}$, and the wheelbase between the second axle and third axle is $L_{3}$. In the six-wheel reverse-phase steering mode, the first axle and the third axle are steering axles, so the inner wheel steering angle is $\alpha_{2}$ or $\alpha_{3}$, and the outer wheel steering angle is $\beta_{2}$ or $\beta_{3}$. And $L_{2}=L_{3}=900 \mathrm{~mm}$, $K=1225 \mathrm{~mm}, \alpha_{2}=\alpha_{3}$, and $\beta_{2}=\beta_{3}$. According to formula (2), the relationship between the $\alpha_{2}$ and $\beta_{2}$ is calculated, and the result is shown in Figure 17. Because of $\alpha_{2}=\alpha_{3}, \beta_{2}=\beta_{3}$, the relationship between $\alpha_{3}$ and $\beta_{3}$ can also be represented by Figure 17 .

In the six-wheel reverse-phase steering mode, the first and third axles are steering axles, and the steering center is on the extension line of the second axle. So, the distance from the steering axle to the horizontal straight line where the steering center is located is $L_{2}$ and $L_{3}$, and $L_{2}=L_{3}=900 \mathrm{~mm}$, so in this mode, $L=L_{2}=L_{3}=900 \mathrm{~mm}$. In this mode, $\alpha_{2}$ and $\alpha_{3}$ are both inner wheel steering angles and $\alpha_{2}=\alpha_{3}$, so in this mode, $\alpha=\alpha_{2}=\alpha_{3}=35^{\circ}$. When the inner wheel steering angle $\alpha$ is the maximum, the steering radius is the minimum, and the maximum of $\alpha$ is $35^{\circ}$. Taking $L=900 \mathrm{~mm}, \alpha=35^{\circ}, \quad K=1225 \mathrm{~mm}$, and $r_{S}=165 \mathrm{~mm}$ into formula (4) can calculate the minimum turning radius, and the minimum steering radius in six-wheel reverse-phase steering mode is about $2832 \mathrm{~mm}$ calculated by formula (4).

It can be seen from the calculation results that when the maximum in-wheel steering angle is $35^{\circ}$, and the minimum turning radius in the six-wheel reversephase steering mode is approximately $763 \mathrm{~mm}$ smaller than that in the front two-wheel steering mode. The main reason is that in the front two-wheel steering mode, only the two wheels of the first axle are responsible for steering. In this mode, the steering center is located in the middle of the rear two axles. So the wheelbase $L$ is bigger in this mode. In the six-wheel reverse-phase steering mode, two wheels of the first axle and two wheels of the third axle are responsible for steering. The steering center is located on the extension line of the second axle, and the wheelbase $L$ is small in this mode. When the wheelbase $L$ of the vehicle is larger, the turning radius of the vehicle is larger. When the wheelbase $L$ of the vehicle is smaller, the turning radius of the 
vehicle is smaller. Therefore, the minimum turning radius in the front two-wheel steering mode is bigger than that in the six-wheel reverse-phase steering mode. The flexibility of the six-wheel reverse-phase steering mode has been improved significantly.

(3) Six-wheel in-phase steering mode: in this mode, the wheels on all three axles are steered, and the steering center is located behind the third axle.

As shown in Figure 18, the steering angle of the inner wheel of the first axle is $\alpha_{4}$, the outer steering angle of the first axle is $\beta_{4}$, the steering angle of the inner wheel of the second axle is $\alpha_{5}$, the outer steering angle of the second axle is $\beta_{5}$, the steering angle of the inner wheel of the third axle is $\alpha_{6}$, the outer steering angle of the third axle is $\beta_{6}$, the lateral distance from steering center to kingpin joint is $X$, the longitudinal distance from the first axle to the steering center is $L_{4}$, the longitudinal distance from the second axle to the steering center is $L_{5}$, and the longitudinal distance from the third axle to the steering center is $L_{6}$. We calculate the relationship between the first, second, and third axle inner wheel steering angles by formula (6). Then, we calculate the steering angle of the outer wheels of each shaft according to formula (2), and the corresponding steering angle relationship is shown in Figure 19. In Figure 18 $L_{4}=3600 \mathrm{~mm}, L_{5}=2700 \mathrm{~mm}, L_{6}=1800 \mathrm{~mm}$, and $K=1225 \mathrm{~mm}$.

The following can be drawn from Figure 18:

$$
\begin{aligned}
& \tan \alpha_{4}=\frac{L_{4}}{X}, \\
& \tan \alpha_{5}=\frac{L_{5}}{X}, \\
& \tan \alpha_{6}=\frac{L_{6}}{X} .
\end{aligned}
$$

Then, there is

$$
\begin{aligned}
& \alpha_{5}=\arctan \left(\frac{3}{4} \tan \alpha_{4}\right), \\
& \alpha_{6}=\arctan \left(\frac{1}{2} \tan \alpha_{4}\right) .
\end{aligned}
$$

As can be seen from Figure 19, the relationship between the inner wheels steering angle of the three axles is fixed, with the change of the steering angle, the proportion of the steering angle of each axle also changes, but it is always stable in a range. The steering ratio of the mechanical steering system is fixed, so it is necessary to choose a more appropriate transmission ratio. According to the angle relationship in Figure 19 and considering the design of the steering gear set, this paper selects the transmission ratio between the three axles as $1: 0.75: 0.50$ in the mechanical six-wheel in- phase steering mode, which can ensure that the three axles steer at the appropriate angle ratio.

(4) Zero-radius steering mode: in this mode, the vehicle has the minimum steering radius, and the steering center of the vehicle is located in the center of the vehicle, that is, at the midpoint of the second axle of the vehicle. At this time, the wheels of the first axle and the third axle steer, and the wheels of the second axle do not steer. And the vehicle revolves around the steering center in the vehicle; only a small circular field is needed to realize the U-turn or steering.

As shown in Figure 20, the wheel diameter is $D$, the tire width is $W$, the radius of the circular space required for steering is $R$, the first axle left wheel steering angle is $n_{1}$, the third axle left wheel steering angle is $n_{2}$, the third axle right wheel steering angle is $n_{3}$, the first axle right wheel steering angle is $n_{4}$, the wheelbase between the first axles and the second axles is $L_{7}=900 \mathrm{~mm}$, the wheelbase between the second axles and the third axles is $L_{8}=900 \mathrm{~mm}$, and the distance between the kingpin joint of the first axle and the steering center is $F$. The distance between two kingpin joints is $M=1225 \mathrm{~mm}$. In this mode, $n_{1}=n_{2}=n_{3}=n_{4}$. The tire specifications selected by this system are 205/50 R16 95H. According to formula (10), the space required for the vehicle's zero-radius steering can be calculated.

To calculate the space required for zero-radius steering, the tire diameter $D$ should be calculated first.

$$
D=\frac{2 W V}{100}+d \times 25.4
$$

Then, according to Figure 20, it is concluded that

$$
\begin{aligned}
& F=\sqrt{L_{7}^{2}+\left(\frac{M}{2}\right)^{2}}, \\
& R=\sqrt{\left(F+r_{S}+\frac{W}{2}\right)^{2}+\left(\frac{D}{2}\right)^{2}}, \\
& R=\sqrt{\left(\sqrt{\left.L_{7}^{2}+\left(\frac{M}{2}\right)^{2}+r_{S}+\frac{W}{2}\right)^{2}+\left(\frac{D}{2}\right)^{2}},\right.} \\
& n_{1}=n_{2}=n_{3}=n_{4}=\arctan \frac{2 L_{7}}{M},
\end{aligned}
$$

where tire aspect ratio $V=50$ and the distance between two kingpin joints $r_{s}=165 \mathrm{~mm}$. We put $L_{7}=900 \mathrm{~mm}$, $L_{8}=900 \mathrm{~mm}, M=1225 \mathrm{~mm}, r_{s}=165 \mathrm{~mm}$, and $V=50$ into formula (10) to calculate the required steering space in this mode. It is calculated that the steering radius required in this mode is about $1390 \mathrm{~mm}$, and the wheel steering angle is $n_{1}=n_{2}=n_{3}=n_{4}=55.76^{\circ}$. Compared with the steering radius of $2834 \mathrm{~mm}$ in the six-wheel reverse-phase steering mode, it is reduced by $1444 \mathrm{~mm}$, and the flexibility of the vehicle is significantly improved [18]. 


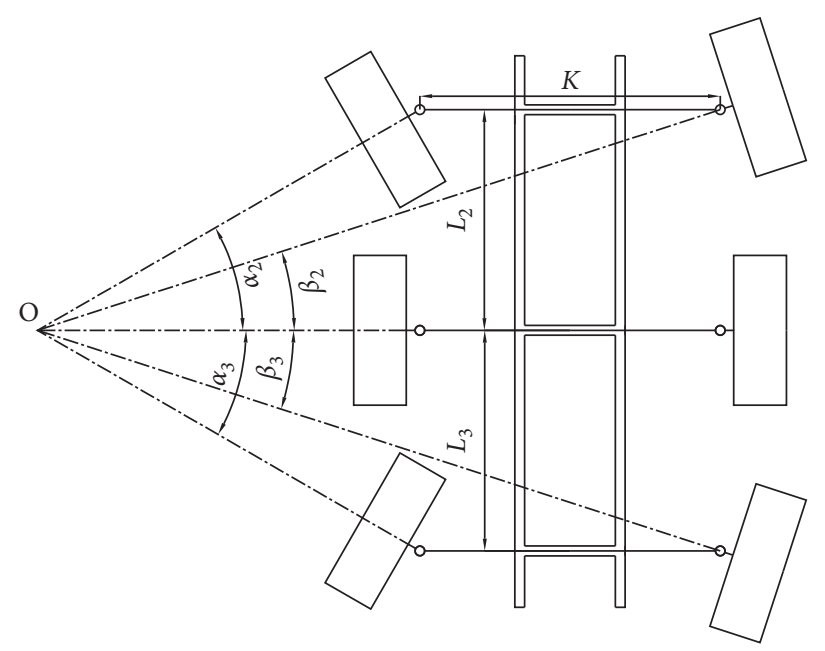

FIgURE 16: Schematic diagram of each wheel angle under the six-wheel reverse-phase steering mode.

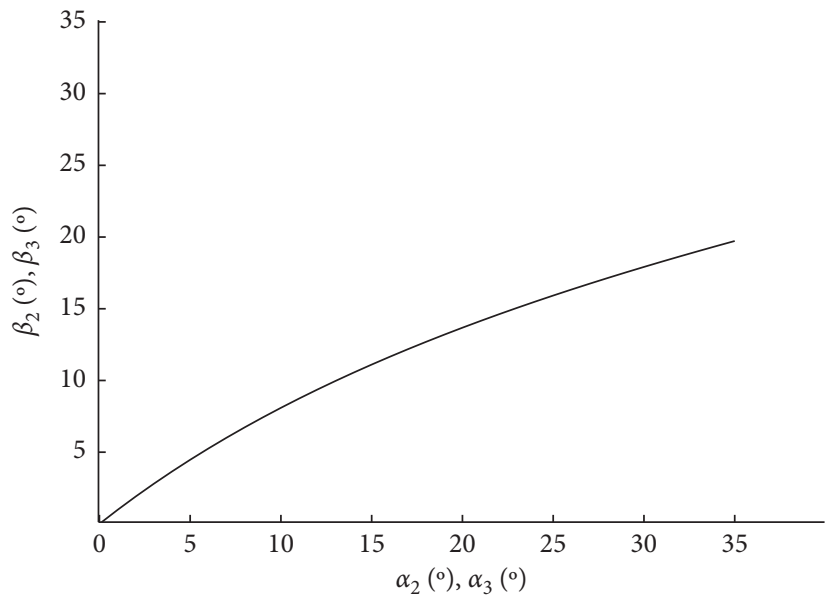

Figure 17: Relationship between $\alpha_{2}\left(\alpha_{3}\right)$ and $\beta_{2}\left(\beta_{3}\right)$.

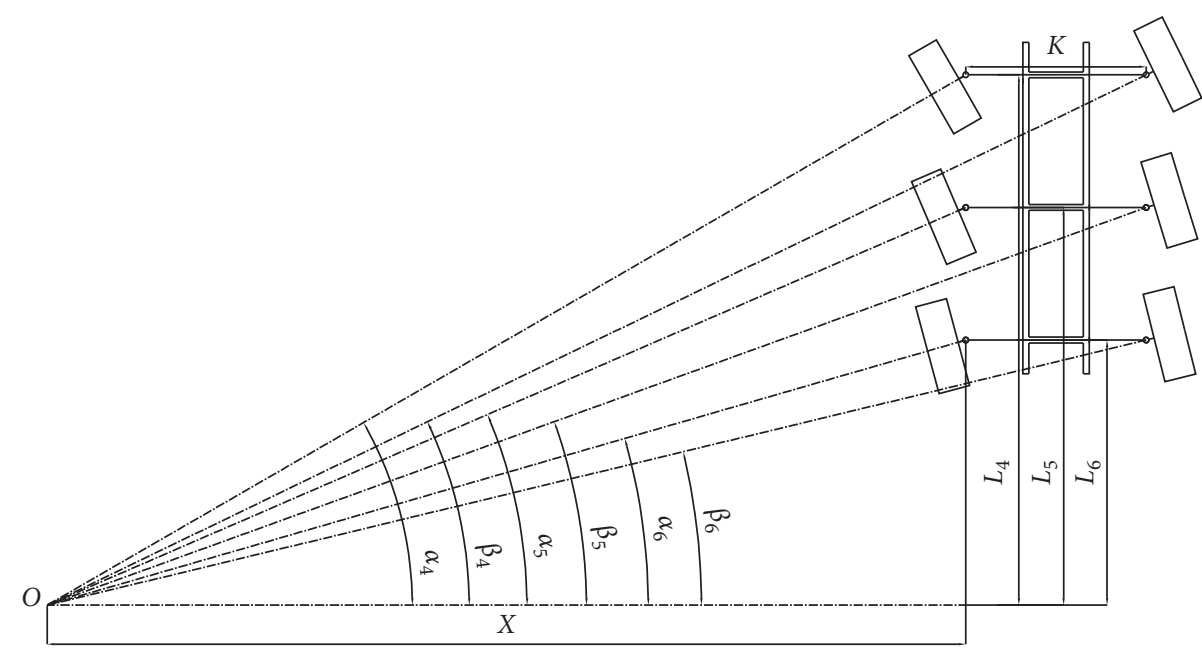

FIGURE 18: Schematic diagram of the steering angle of each wheel in the six-wheel in-phase steering mode. 
6.2. Kinematics Analysis of the Steering Trapezoid. The vehicle matched with the steering system is an off-road vehicle, and the design of the steering system is more inclined to the off-road performance. The six-wheel reverse-phase steering is more suitable for off-road conditions. Therefore, the steering trapezoid is designed according to the theoretical Ackerman steering angle of six-wheel reverse-phase steering [19]. The suspension of the vehicle matched with this steering system adopts independent suspension, so a segmented steering trapezoid mechanism is needed. The kinematics model of steering trapezoid is shown in Figure 21.

According to experience and vehicle parameters, the parameters of the steering trapezoid of this system are selected [20], the software Solidworks is used for simulation, and the parameters are adjusted. The parameters of the steering trapezoid are finally determined as shown in Table 1.

The Ackerman ratio is the ratio of the actual angle difference between the inner and outer wheels and the theoretical angle difference between the inner and outer wheels. According to different situations, the steering Ackerman ratio will affect the tire wear and steering return performance, so it is necessary to calculate the steering Ackerman ratio. According to the actual outer wheel angle and formula (12), the steering trapezoid is verified, and the Ackerman ratio satisfaction of the steering system is shown in Table 2.

$$
R_{A C}=\frac{\theta_{i}-\theta_{o r}}{\theta_{i}-\theta_{o}} \times 100 \% .
$$

Here, $R_{A C}$ is the Ackerman rate, $\theta_{i}$ is the inner wheel steer angle, $\theta_{\text {or }}$ is the actual outer wheel steer angle, and $\theta_{o}$ is the Ackerman theoretical outer wheel steer angle.

The Ackerman rate is $100 \%$ for standard Ackerman steering because the tire is a nonrigid body, the steering performance of the vehicle in the standard Ackerman steering mode is not the best, and the tire wear is more serious. Generally speaking, when the inner wheel angle is less than $20^{\circ}$, the Ackerman rate is more appropriate in the range of $40-60$. And when the inner wheel angle is $20^{\circ}$ to $35^{\circ}$, the Ackerman rate is more appropriate in the range of 60-80. From the data in Table 2, it can be seen that the Ackerman rate of the mechanical steering system meets the requirements, and the performance of the steering trapezoid is better.

The steering controller controls the rotation angle of the steering motor. The steering motor drives the lateral movement of the D-type screw, and the lateral movement of the D-type screw drives the tie rod, which in turn drives the wheels to steer. In the transmission process, the distance between the motor rotation angle and the lateral movement of the D-type screw has a fixed transmission ratio. However, there is no fixed transmission ratio between the moving distance of the D-type screw and the wheel steer angle. It is necessary to calculate the relationship between the moving distance of the D-type screw and the wheel steer angle so that the motor can be controlled more quickly during the control process. Combining the parameters of the steering trapezoid, the relationship between the moving distance of the D-type screw and the wheel steer angle is analyzed. The kinematics model is shown in Figure 22.

The parameters of the steering trapezoid are shown in Table 1. According to the parameters of the steering trapezoid, the relationship between $S$ and $\theta$ can be derived, and the relationship is shown in

$$
\theta_{3}=\theta_{1}-\arctan \frac{t_{1}+t_{2}-t_{3}}{t_{4}+\sqrt{t_{5}^{2}-\left(t_{2}-t_{3}\right)^{2}}},
$$

where

$$
\begin{aligned}
& t_{1}=2\left[4 h^{2}+4(b+c+s)^{2}\right] h \\
& t_{2}=\sqrt{4\left[4 h^{2}+4(b+c+s)^{2}\right]\left[\left(h^{2}+(a+b+s)\right)^{2}+l_{2}^{2}-l_{1}^{2}-4(b+c+s)^{2} l_{2}^{2}\right]} \\
& t_{3}=4 h\left[h^{2}+(a+b+S)^{2}+l_{2}^{2}-l_{1}^{2}\right] \\
& t_{4}=2(b+c+s)\left[4 h^{2}+4(b+c+s)^{2}\right] \\
& t_{5}=2 l_{2}\left[4 h^{2}+4(b+c+s)^{2}\right]
\end{aligned}
$$

where $b$ and $c$ are as follows:

$$
\begin{aligned}
& b=\cos \theta_{1}, \\
& c=\sqrt{l_{2}^{2}-\left(l_{1} \sin \theta_{3}-h\right)^{2}} .
\end{aligned}
$$

As shown in Figure 22, taking the left half of the steering trapezoid as an example, the D-type screw moves to the left to drive the wheels to turn right, and the D-type screw to move to the right drives the wheels to turn to the left. The left wheel can turn right up to $90^{\circ}$, the left turn can turn left up to $35^{\circ}$ (the right wheel is the opposite), and the value range of $\theta_{3}$ is $-35^{\circ}$ to $90^{\circ}$. In order to distinguish the moving direction of the $\mathrm{D}$-type screw and the steering direction of the wheels, it is assumed that the left shift of the D-type screw is negative and the right shift is positive, and the left steer of the wheel is 


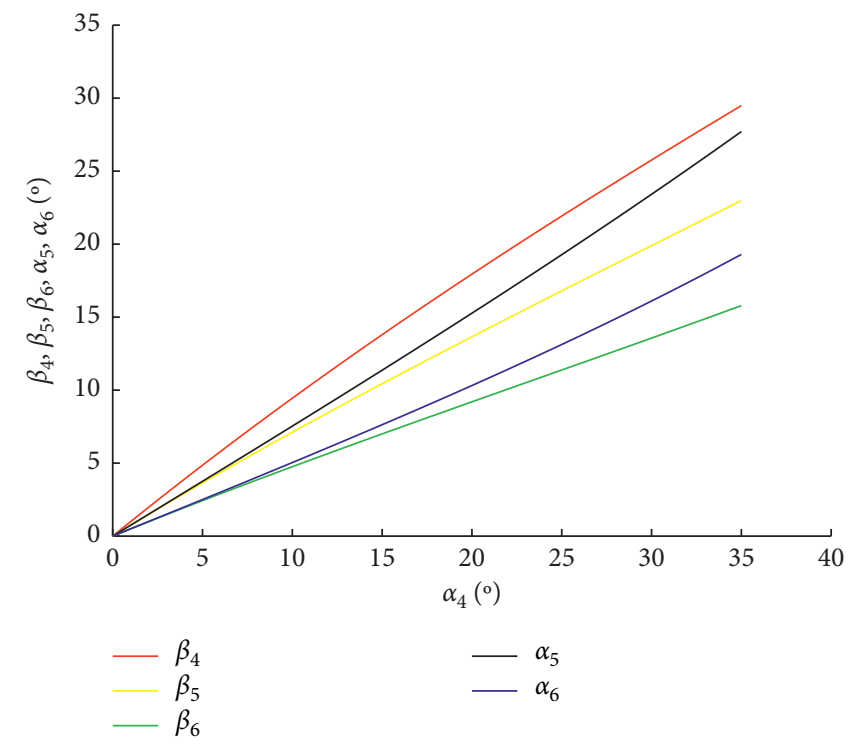

FIGURE 19: The relationship between the steering angle of each wheel and the internal rotation angle of the first axles in the six-wheel inphase steering mode.

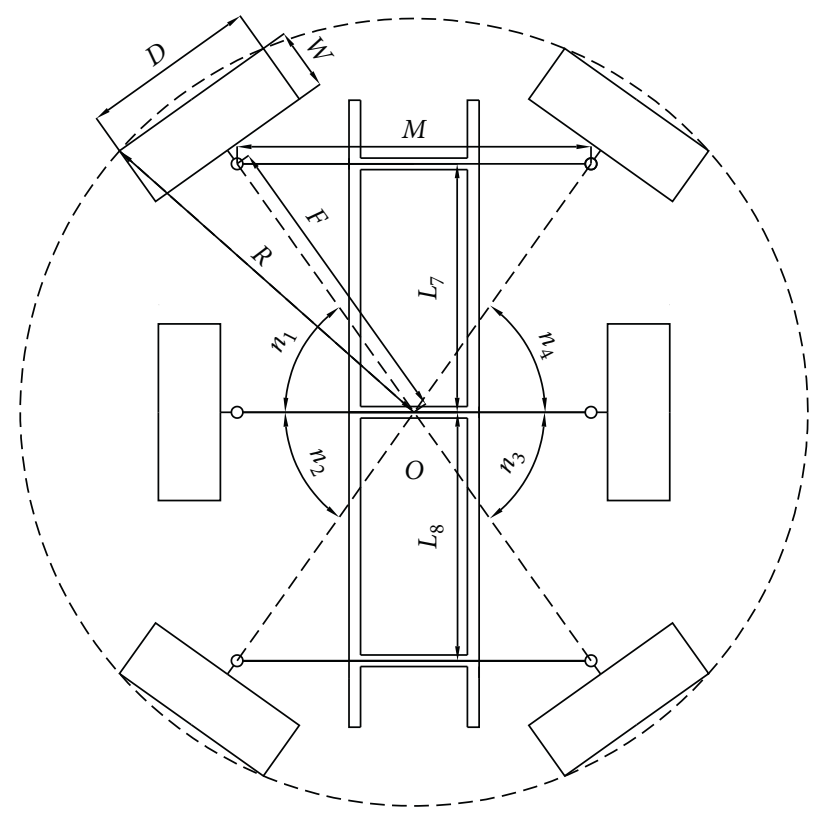

FIGURE 20: Schematic diagram of zero-radius steering.

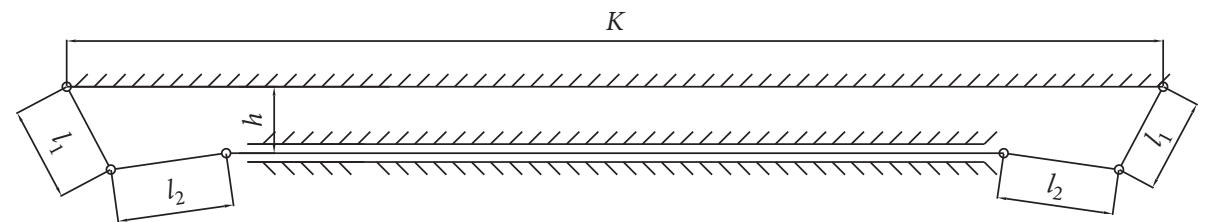

FIGURE 21: Steering trapezoid kinematics model.

negative and the right steer is positive. We put the value range of $\theta_{3}$ into formula (13) to obtain the relationship between $s$ and $\theta_{3}$ as shown in Figure 23.
According to Figure 23, it can be seen that $S$ and $\theta_{3}$ are not proportional. When the steering motor drives the steering wheel to steer, the motor angle can be quickly 
TABLE 1: Steering trapezoid parameters.

Parameter name

Numerical value $(\mathrm{mm})$

Distance between two kingpin joints $(K)$

1225.00

Length of steering arm $\left(l_{1}\right)$

105.00

Length of the tie rod $\left(l_{2}\right)$

130.00

Distance of steering mechanism and the first axle $(h)$

74.41

The universal joint of the steering mechanism $(M)$

869.00

TABLE 2: Check calculation of the Ackerman rate.

\begin{tabular}{lccc}
\hline$\theta_{i}\left({ }^{\circ}\right)$ & $\theta_{o}\left({ }^{\circ}\right)$ & $\theta_{\text {or }}\left({ }^{\circ}\right)$ & $R_{A C}$ \\
\hline 1 & 0.98 & 0.99 & 50 \\
5 & 4.47 & 4.77 & 43 \\
10 & 8.09 & 9.09 & 48 \\
15 & 11.11 & 12.96 & 52 \\
20 & 13.68 & 16.36 & 58 \\
25 & 15.92 & 19.24 & 63 \\
30 & 17.92 & 21.54 & 70 \\
35 & 19.72 & 23.20 & 77 \\
\hline
\end{tabular}

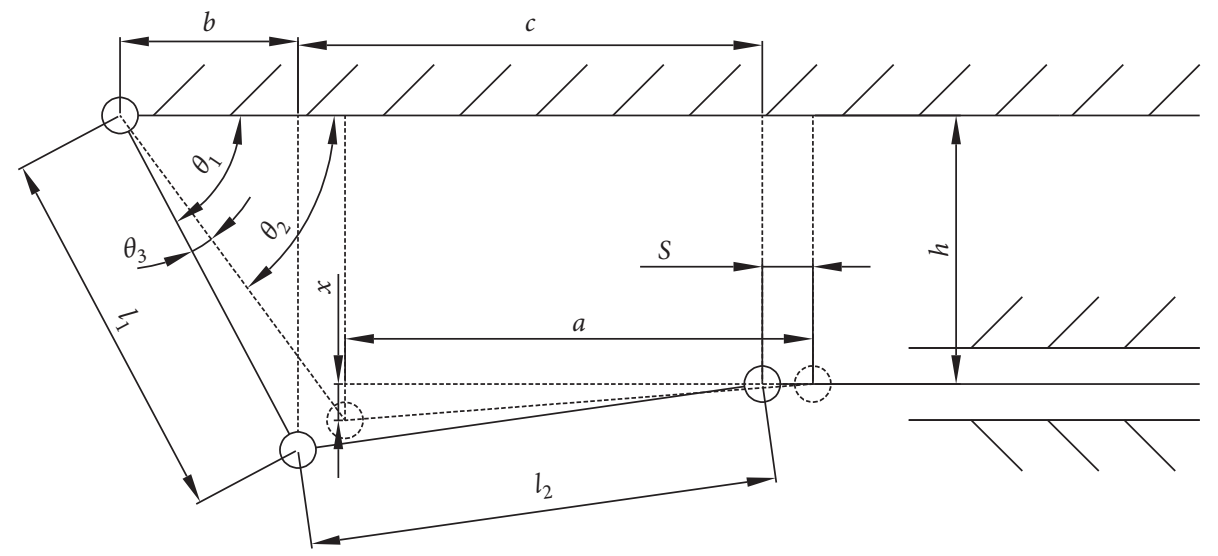

FIGURE 22: One-sided steering kinematics model.

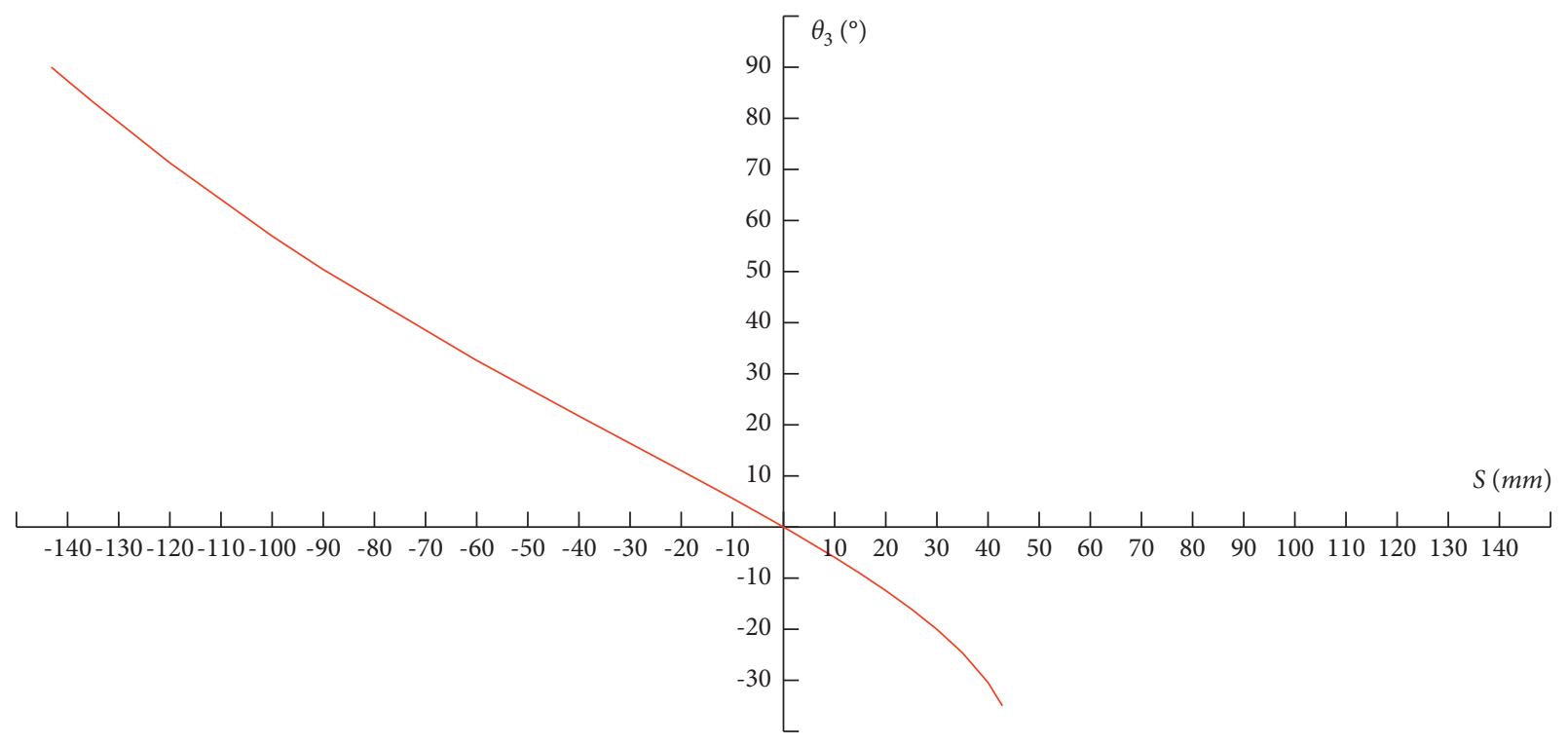

Figure 23: The relationship between $S$ and $\theta_{3}$. 
controlled according to this diagram, and then, the angle of steer is corrected according to the angle signal fed back by the wheel angle sensor, so that the wheel angle can be controlled quickly and accurately.

\section{Conclusions}

The steering system is one of the key subsystems of the vehicle. A steering system with good performance is the guarantee for the safe operation of the vehicle. Six-wheeled off-road vehicles need to pass complex roads. A good performance steering system can improve the passing and flexibility of the vehicle. Therefore, this paper studies the steering system of three-axle six-wheeled vehicles. And the steering system of a three-axle six-wheeled vehicle is designed, modeled, and simulated. The main research contents and conclusions of this paper are as follows:

(1) Combining the design goals of the off-road vehicle, the steering mode and the application scenarios of the steering mode are established. Considering the application scenarios of off-road vehicles, the steering mode of the steering system is designed, and the specific application scenarios of the steering mode are introduced. Since the vehicle has five steering modes, the vehicle can adapt to more usage scenarios. For example, the application scenarios of lateral parking, zero-radius steering, and six-wheel reverse-phase steering can significantly improve the flexibility of the vehicle. It can be seen that the performance of the steering system is superior.

(2) The structure of the system and the working principle of each main component are analyzed combined with the CAD model. Among them, the longitudinal linkage rack is the key to the mechanical steering system, which can realize the linkage of the three steering mechanisms on three axles and the switching of the steering mode. The sliding base is the key part of the combination of mechanical steering and electronic control steering. Through the design of the structure, the ingenious combination of mechanical steering and electronic control steering is realized, and the performance of the steering system is improved.

(3) The hardware and control method of the electronic control system are combined to realize the control of the electronic control steering system. When controlling the wheel angle, the wheel angle controller determines the target angle of other wheels according to the input front inner wheel angle signal, and then, the wheel angle controller controls each steering motor according to the target angle of each wheel. Then, through the feedback of the angle sensor to correct the wheel angle to form a closedloop control, this can control the wheel angle more quickly and accurately.

(4) The construction of the kinematics model and the calculation of related data were carried out. The calculation results showed that the six-wheel inphase steering can significantly reduce the steering radius, and the zero-radius steering can also achieve U-turn steering in a narrow space. This will ensure the flexibility of the vehicle. Through the calculation of the steering trapezoid, it is found that the design of the steering trapezoid can better meet the Ackerman rate, and the steering trapezoid has good performance.

\section{Data Availability}

The data used to support the findings of this study are included within the article.

\section{Conflicts of Interest}

The authors declare that they have no conflicts of interest.

\section{Authors' Contributions}

Methodology and original draft preparation were done by Haixiang Bu; three-dimensional modeling and analysis were contributed by Aijuan Li and Wei Li; reviewing and editing were performed by Xin Huang and Jian Wang. All authors have read and agreed to the published version of the manuscript.

\section{Acknowledgments}

This work was supported by National Natural Science Foundation of China (Grant Nos. 51505258, 61601265, and 51405272), Shandong Provincial Natural Science Foundation, China (Grant Nos. ZR2015EL019, ZR2020ME126, and ZR2021MF131), Shandong Province Higher Educational Youth Innovation Science and Technology Program (Grant Nos. 2019KJB019 and 2020KJN002), China Postdoctoral Science Foundation (Grant No. 2021M701405), Open Project of State Key Laboratory of Mechanical Behavior and System Safety of Traffic Engineering Structures, China (Grant no. 1903), and Open Project of Hebei Traffic Safety and Control Key Laboratory, China (Grant no. JTKY2019002).

\section{References}

[1] Z. Zhang, X. Zhang, H. Pan et al., "A novel steering system for a space-saving 4WS4WD electric vehicle: design, modeling, and road tests," IEEE Transactions on Intelligent Transportation Systems, vol. 18, no. 1, pp. 114-127, 2017.

[2] Z. Yang, G. Li, H. He, and G. Li, "Study on road feeling simulation control algorithm for four-wheel independent drive and steering electric vehicle," in Proceedings of the 2017 Chinese Automation Congress (CAC), pp. 4872-4875, Jinan, China, October 2017.

[3] H.-J. Yan, D.-B. Luo, and L.-F. Qi, "Research on the omnidirectional steering system of patrol vehicles in urban utility tunnels," Machine Design and Manufacture, vol. 1, pp. 159-162, 2021. 
[4] L. Wang, "Research on four-axis mechanical linkage steering mechanism based on single steering tie-rod," Coal Mine Machinery, vol. 41, no. 2, pp. 53-56, 2020.

[5] A. N. Kazak and D. M. Filippov, "Development of in-wheel motor for vehicles," in Proceedings of the 2019 IEEE Conference of Russian Young Researchers in Electrical and Electronic Engineering (EIConRus), pp. 1406-1408, Moscow, Russia, January 2019.

[6] Y. Cao and M. Qiao, "Application of fuzzy control in four wheel steering control system," in Proceedings of the 2017 International Conference on Advanced Mechatronic Systems (ICAMechS), pp. 62-66, Xiamen, China, December 2017.

[7] R. Prasad and Y. Ma, "Hierarchical control coordination strategy of six wheeled independent drive (6WID) skid steering vehicle," IFAC-PapersOnLine, vol. 52, no. 5, pp. 60-65, 2019.

[8] F. Du, Z. Guan, D. Su, and J. He, "Control strategy for fourwheel steering vehicle based on collaborative simulation," in Proceedings of the 2017 9th International Conference on Modelling, Identification and Control (ICMIC), pp. 348-353, Kunming, China, July 2017.

[9] J. Wang, G. Tan, and C. Sun, "Research on vehicle four-wheel steering based on model-free adaptive control," in Proceedings of the 2020 5th International Conference on Electromechanical Control Technology and Transportation (ICECTT), pp. 372-376, Nanchang, China, May 2020.

[10] Y. Ye, L. He, and Q. Zhang, "Steering control strategies for a four-wheel-independent-steering bin managing robot," IFAC-PapersOnLine, vol. 49, no. 16, pp. 39-44, 2016.

[11] G. S. G. Ravikanth and C. Sujatha, "Dynamic modeling and simulation of a three-wheeled hub motor vehicle," in Proceedings of the 2017 IEEE Transportation Electrification Conference (ITEC-India), pp. 1-5, Pune, India, December 2017.

[12] Y. Zhao, G. Tao, J. Li, X. Zhang, and J. Gong, "Dynamic model and predictive control of electric driven eight-wheeled differential steering autonomous vehicle," in Proceedings of the 2020 3rd International Conference on Unmanned Systems (ICUS), pp. 412-417, Harbin, China, November 2020.

[13] M. Muenster, M. Lehner, and D. Rixen, "Requirement derivation of vehicle steering using mechanical four-poles in the presence of nonlinearities," Mechanical Systems and Signal Processing, vol. 155, 2021.

[14] S. Yim, "Comparison among active front, front independent, 4-wheel and 4-wheel independent steering systems for vehicle stability control," Electronics, vol. 9, no. 5, 2020.

[15] F. Xu, X. Liu, W. Chen, and C. Zhou, "Dynamic switch control of steering modes for four wheel independent steering rescue vehicle," IEEE Access, vol. 7, pp. 135595-135605, 2019.

[16] Z. M. U. Din, W. Razzaq, U. Arif, W. Ahmad, and W. Muhammad, "Real time Ackerman steering angle control for self-driving car autonomous navigation," in Proceedings of the 2019 4th International Conference on Emerging Trends in Engineering, Sciences and Technology (ICEEST), pp. 1-4, Karachi, Pakistan, December 2019.

[17] Q. Qiu, Z. Fan, Z. Meng et al., "Extended Ackerman steering principle for the coordinated movement control of a four wheel drive agricultural mobile robot," Computers and Electronics in Agriculture, vol. 152, pp. 40-50, 2018.

[18] S. S. Singh and G. Singh Mavi, "Optimization and mathematical calculation of turning radius for 4 wheel steering systems in maruti suzuki baleno," International Journal of Innovative Technology and Exploring Engineering (IJITEE), vol. 8, no. 11, pp. 3961-3963, 2019.
[19] D. Wei, Y. Wang, T. Jiang, S. Zheng, W. Zhao, and Z. Pan, "Chaos vibration of pinion and rack steering trapezoidal mechanism containing two clearances," Mechanical Systems and Signal Processing, vol. 92, pp. 146-155, 2017.

[20] G. R. Kiranchand, T. Soni, and A. C. Mitra, "Experimental design, sensitivity analysis of steering geometry and suspension parameters," Materials Today: Proceedings, vol. 5, no. 2, pp. 5743-5756, 2018. 